\title{
Mapping the Human Connectome
}

\author{
Arthur W. Toga, Ph.D., Kristi A. Clark, Ph.D., Paul M. Thompson, Ph.D., David W. Shattuck, \\ Ph.D., and John Darrell Van Horn, Ph.D. \\ Laboratory of Neuro Imaging (LONI), Department of Neurology, David Geffen School of Medicine, \\ University of California Los Angeles, 635 Charles E. Young Drive SW, Suite 225, Los Angeles, \\ CA 90095-7334, (310) 206-2101 (phone), (310) 206-5518 (fax) \\ Arthur W. Toga: toga@loni.ucla.edu
}

\section{Introduction}

The human nervous system consists of on the order of one hundred billion neurons that are interconnected to form a relatively small number of functional neural networks that underlie behavior and cognition. The elemental beauty of this system was elegantly described in the work of Ramón y Cajal and others well over a century ago. Since that time, despite the intense effort that has gone into elucidating the structure and function of neural systems, we do not currently have a comprehensive map of the complete network connectivity structure of the brain of any species, with the notable exception of a worm, Caenorhabditis elegans. ${ }^{1}$ In humans, our basic understanding of network connectivity is largely based on painstaking neuroanatomical efforts conducted at a microscopic scale. ${ }^{2,3}$ These maps are derived histologically, and are sparsely observed and incomplete.

The connection matrix of the human brain, i.e., the human "connectome", represents an indispensable foundation for basic and applied neurobiological research. The axon of a neuron in one region of the brain extends to another region following a particular anatomic course or trajectory. The ensemble over all brain neurons of axonal origin, termination, and trajectory relative to other structures defines the connectome, at least from an anatomical point of view.

Functional networks are certainly served by these anatomical substrates but may involve multiple overlapping systems carrying quantifiably different forms of information to other parts of the brain for integration, further processing, and resulting behavioral action. Brain function depends on the communication among neurons organized within local as well as widely distributed circuits, leading to a vast and extraordinarily complicated set of interconnected brain systems. Human connectomics explores the structural and functional organization and properties of these neural connections to define the architecture of the brain.

For the foreseeable future, a comprehensive description of the complete connectome of even a single human brain might be viewed as unattainable. But the science of connectomics is devoted to filling in the gaps, with a variety of imaging and other modalities. Building on traditional neuroscience techniques, the Human Connectome Project (HCP) (http:// www.humanconnectomeproject.org/) uses novel imaging technologies and mathematical analysis methods and databases to organize, relate, and share the derived information. With support from the NIH Blueprint program (http://neuroscienceblueprint.nih.gov/), researchers at multiple institutions are rapidly performing the physical, data processing, informatics, and inferential challenges of conducting human connectomics research. This multi-site effort, in concert with similar efforts elsewhere, seeks to obtain connectomic data sets and make them 
openly available for the expert and lay public to explore and examine using conventional web browsers, or to study them in greater detail using more advanced interactive tools.

\section{A map of the Human Connectome}

The connectome may be defined as the complete, point-to-point spatial connectivity of neural pathways in the brain. ${ }^{4}$ This detailed, multiscaled, and multivariate matrix is defined computationally and statistically using sophisticated in vivo neuroimaging data, electrical recordings, and postmortem tissue samples to provide a detailed framework to understand the anatomically based interactions of functional regions of the brain. The connectome gives rise to population-level atlases of distributed connectivity and makes it possible to assess disruptions of connectivity in clinical samples. Demographic, genomic, and cognitive/ behavioral data can be superimposed on the connectome to permit inferences concerning genetic and other influences on connectedness. ${ }^{5,}{ }^{6}$ Information concerning connectivity is essential for understanding fundamental cognitive operations, systems-level brain activity, conditional structure-function models of brain, and debilitating brain diseases.

Mapping the functional and structural connectivity of the brain using the latest neuroimaging methods must be accompanied by the tools needed to explore those data and to appreciate their richness. The expectation is that HCP will (1) have immediate impact on the field of neuroscience and beyond; (2) provide a framework and set of tools with enduring utility and value; and (3) enable the broad community of investigators to generate and test new hypotheses based on the HCP data corpus and summary maps, thereby informing and advancing their own research priorities.

\section{Methods}

Historically, most neuroimaging studies of the human brain have employed a modular view of the brain, e.g., region $\mathrm{X}$ is responsible for function $\mathrm{Y}$. This modularity of mind approach, however, is insufficient for describing the vast set of cognitive and behavioral operations of which the brain is capable. A more appropriate approach considers which network of two or more connected and interacting regions are employed for a given function. It has not always been possible to view networks in the brain; it was not until recently that any magnetic resonance imaging (MRI) sequence was capable of discerning individual axon bundles.

Traditional anatomical acquisitions used scanning protocols designed to exploit the differences in $\mathrm{T} 1$ and $\mathrm{T} 2$ relaxation times between fat and water, as well as the proton density of tissues. These sequences are ideal for measuring the boundaries between gray matter, white matter, and cerebrospinal fluid (CSF), as white matter has the highest fat content and CSF has the highest water content. However, in these scans, the brain's white matter is merely seen as an undifferentiated, homogeneous mass. Other types of scanning protocols may be used to measure brain chemistry, e.g., MR spectroscopy, as well as cerebral blood flow, e.g., arterial spin labeling, and even changes in cerebral blood oxygenation levels, e.g., BOLD fMRI.

The development of MRI methods sensitive to water diffusion made it possible to reconstruct neural fiber pathways in vivo. While most commonly available 1.5 and 3 Tesla MRI systems can perform such data acquisition, new scanners, with more advanced gradient capabilities, special acquisition sequences, and multi-channel head coils, can improve resolution and increase the amount of connectomic information acquired from any given subject. Our initial work at high magnetic fields, performing diffusion imaging on 7-Tesla scanners, ${ }^{7}$ shows that the enhanced signal-to-noise ratio can be used to more accurately measure properties of white matter fibers between two or more regions and, therefore, better characterize the process of information exchange between them. 


\section{Diffusion Imaging}

Diffusion magnetic resonance imaging (dMRI) measures the directional diffusion of water molecules in tissue, thus enabling the mapping of the underlying white matter structure of the living human brain. ${ }^{8-10}$ Early diffusion imaging experiments by Moseley, ${ }^{11}$ Beaulieu, ${ }^{9}$ and others demonstrated that, within the nervous system, greater diffusion was observed when the relative orientation of the diffusion-sensitizing gradient was parallel to axons (whether myelinated or not) than when it was perpendicular. This fortuitous discovery has now resulted in diffusion imaging data being acquired as a matter of course in many human neuroimaging studies. Initially limited to estimating 6-parameter diffusion tensors, more sophisticated diffusion imaging approaches have been introduced to examine many more potential diffusion directions, such as in High Angular Resolution Diffusion Imaging (HARDI), or to extract the results of examining the spectral aspects of diffusion, such as in Diffusion Spectrum Imaging DSI ${ }^{12}$; or hybrid diffusion imaging $\left(\mathrm{HYDI}^{7}\right)$. Today there are many variations on acquisition and analysis techniques employed to map the underlying trajectories of fascicles using dMRI, with more appearing on the horizon. A significant line of work is studying how the recovered brain networks depend on the spatial and angular resolution of the scans ${ }^{13}$ and how the choice of scanning protocol affects the reproducibility and reliability of the connections and computed networks. ${ }^{14}$

\section{Tractography}

Estimates from dMRI of how gray matter regions are connected to each other via white matter bundles typically occurs in two steps: (i) an estimation of the orientation of diffusion within each voxel and (ii) an estimation of fiber trajectories across voxels by means of a tractography algorithm. Tractography - the computational process of tracking the path of connectivity through the brain - can identify and help quantify the white matter structural integrity of the in vivo human brain. ${ }^{15}$ However, such methods must contend with the fact that the spatial scale of voxels is on the order of millimeters, while the spatial scale of axonal diameters is on the order of microns. Therefore, dMRI is currently a macroscale estimate of the trajectories of neural pathways. But once obtained, the results can be readily visualized in $3 \mathrm{D}$ and coded by the orientation of diffusion to render some of the most compelling images of the human brain we presently have. ${ }^{16}$

Several methods exist for creating these matrices of inter-regional connectivity. The simplest form of fiber tractography is deterministic streamline fiber tracking. ${ }^{1-19}$ In this method, the within-voxel fiber orientation distribution (FOD), or even just the principal eigenvector or dominant direction of diffusion from DTI, is used to track major white matter tracts in the brain in a point-by-point fashion. While this method is computationally simple and intuitive, there are several known disadvantages, such as decreased accuracy with distance from the region of interest (ROI), dependence on fractional anisotropy (FA) and turning angle thresholds, and errors in voxels with partial volume effects. There is also no inherent indication of the confidence or uncertainty for each reconstructed trajectory, although the latter can be remedied using bootstrapping techniques. ${ }^{20}$ Some of the newer tractography algorithms are probabilistic ${ }^{21}$ and show promise in overcoming some of the disadvantages of deterministic methods. Whole-brain tractography may also be performed, seeding fibers at all possible voxels in the brain. Some neuroanatomically informed algorithms aim to cluster the resulting fibers into anatomically meaningful bundles that correspond to the nomenclature recognized by anatomists.

A comprehensive map of the human brain requires the identification and labeling of tracts both large and small. Manual protocols have been developed to identify large white matter tracts. ${ }^{22,23}$ An alternative to manual protocols is to use one of many existing atlases, e.g. the 
LBP40 atlas ${ }^{24}$, the AAL atlas ${ }^{25}$, the Jülich histological atlases ${ }^{26}$, the Johns-Hopkins DTIbased atlases ${ }^{27}$, to form seed points.

Work to automate such procedures is an active area of computational research. Several fiber clustering algorithms can identify tracts of various sizes. ${ }^{28-30}$ Given a set of fiber tracts, spectral clustering first projects them into an embedding space and then performs standard clustering in this space. ${ }^{31,32}$ Compared to other fiber clustering algorithms, ${ }^{28,30,33}$ spectral clustering captures the intrinsic relation of fiber bundles and is robust to pose variations (cf. the spectral modeling of intrinsic geometry in 3D shape analysis ${ }^{34-36}$ ). Spectral clustering techniques are straightforward to implement on many computer systems as they only involve matrix-based computations, but given the number of them being performed, this can necessitate the use of large-scale CPU architectures. Even so, without including anatomical information explicitly, the resulting tracts may not match anatomical expectations. Newer methods can combine atlases with pattern recognition methods, to automatically group fibers into tracts, avoiding the labor-intensive task of interactive manual labeling. ${ }^{37,38}$

\section{Networks in the Connectome}

Once structural connectivity has been mapped for the brain, properties of the resulting networks can be studied by adapting some of the core constructs from graph theory. ${ }^{39}, 40 \mathrm{In}$ graph theory, a graph is any representation of a network in terms of a set of nodes and connections through which the nodes interact. In the human brain, gray matter regions may be treated as nodes and the axonal bundles, which can be estimated from dMRI, form the connections of the graph. Networks are often classified according to two metrics: a clustering coefficient $(\mathrm{C})$ which measures how connected each node is, and the characteristic path length $(\lambda)$, which measures the average distance between nodes, where $\lambda$ is inversely related to network efficiency. We note that the physical length of the connection is not considered by these metrics, only the number of network nodes that must be traversed to go from one point in the network to another. For many years, networks were classified as either regular (high C and high $\lambda$ ) or random (low $C$ and low $\lambda$ ). In 1998, it was observed that many non-biological networks, such as networks of film actors or power grids, did not fit well into either of these categories. Therefore, a new class of network, called the "small world" network, was introduced as having a high $\mathrm{C}$, like regular networks, and a low $\lambda$, like random networks. ${ }^{41}$ The rationale for examining "small worldness" in the brain using neuroimaging-derived connectivity matrices is that it is possible to measure the capacity of the cortex to process information. ${ }^{42}$

Recently, this small world network modeling approach has been applied to the study of structural and functional connectivity of the human brain because it seems to capture many relevant features, such as high local clustering (cortical regions) combined with longdistance connections between clusters (large white matter tracts). ${ }^{40}$ Additionally, two BOLD fMRI studies have demonstrated that the efficiency of the human cortex is correlated with intelligence quotient (IQ) such that higher IQ is associated with a more efficient cortical network, i.e., those having a "shorter" $\lambda .{ }^{31,43}$ These methods have also been applied to large populations of subjects to demonstrate that the overall efficiency of the brain decreases with age ${ }^{44}$ and changes during development. ${ }^{45}$ Additionally, graph theory constructs have been used to reveal changes in the neuroanatomical networks of subjects blind from an early age $^{46}$ and changes in the functional networks of attention-deficit hyperactivity disorder (ADHD) subjects. ${ }^{47}$ Indeed, the mapping of the properties, organization, and structure of brain networks will require that new network theoretical metrics be developed as we extract still finer-grained elements worthy of characterization for healthy as well as diseased or damaged networks. 
The large-scale analysis of anatomical networks has already yielded surprising discoveries, including differences in network properties between the left and right hemispheres. ${ }^{48}$ Our studies of DTI scans from over 700 twins show that the density and integrity of brain connections is under strong genetic control. ${ }^{49-51}$ The first genome-wide scans of the connectome have discovered specific variants on the human genome that affect brain connectivity. ${ }^{5}$ Healthy carriers of autism risk genes, such as a highly prevalent variant in CNTNAP2, have altered brain connectivity that may shed light on the brain dysfunction in autism. ${ }^{52}$ Large scale normative data on developing brain connectivity show characteristic changes in network efficiency and small-world properties between ages 12 and $30 .{ }^{45}$ There are also clear clinical applications. One study showed that small-world measures of anatomical networks, computed from DTI, predicted future decline in people with mild cognitive impairment MCI. ${ }^{53}$

While the activities of the HCP and other efforts indicate the remarkable promise to map human connectivity with brain imaging, simply collecting a large number of data sets at high-resolution on sophisticated scanners will not be sufficient to provide the necessary understanding of brain circuitry. In the same way as sequencing the human genome was only the first step toward mapping functions to specific genes, much work will be needed to transform DTI/DSI/HARDI data into connectivity atlases. Unfortunately, this is not as simple as just "taking averages". For instance, imagine having a patient for whom a complete map of connectivity has been constructed from a well-understood regional brain parcellation scheme; one wishes to compare this map to that of another patient who has experienced brain trauma affecting several brain regions. Can these two networks, and the metrics derived from them, be directly compared? Though seemingly simple, there are many mathematical challenges in comparing such networks or even pooling network metrics across differing individuals: how to "align" the network graphs, how to compare existing and missing node locations, and how to identify whether networks are the same or, in fact, are different altogether. Quantifying how these networks are strengthening in children as they develop and declining in adults as they age is only just beginning to be examined. ${ }^{45,53}$ Moreover, making inferences on the relative functional roles of many putative connections will require that cognitive task paradigms be refined or invented to tap into the specific functional importance of region-to-region connectedness. Furthermore, information regarding the direction of information transmission is unobtainable with diffusion imaging alone, and it is difficult to estimate with fMRI where the speed of neural transmission is many hundreds of times faster than the time scale of sampling the scans. Even then, the true measure of network capacity will only be achieved once structural and functional properties of the network can be mutually assessed. Such computational challenges are not trivial and will necessitate new thinking concerning what we mean by population-level "average" networks and what deviations from this average mean.

\section{Summary}

Knowledge of the properties of white matter fiber tracts is a crucial and necessary step toward a precise understanding of the functional architecture of the living human brain. Previously, this knowledge was severely limited, as it was difficult to visualize these structures or measure their functions in vivo. The Human Connectome Project has recently generated considerable interest because of its potential to explore connectivity and its relationship with genetics and behavior. For neuroscientists and the lay public alike, the ability to assess, measure, and explore this wealth of layered information concerning how the brain is wired is a much sought after prize.

The navigation of the human connectome and the discovery of how it is affected through genetics, and in a range of neurological and psychiatric diseases, have far reaching 
implications. From a range of ongoing connectomics related activities, the systematic characterization of brain connectedness and the resulting functional aspects of such connectivity will not only realize the work of Ramón y Cajal and others, but will also greatly expand our understanding of the brain, the mind, and what it is to be truly human. The similarities and differences that mark normal diversity will help us to understand variation among people and set the stage to chart genetic influences on typical brain development and decline during aging. What is more, an understanding of how brains might become disordered will shed light on autism, schizophrenia, Alzheimer's, and other diseases that exact a tremendous and terrible social and economic toll.

\section{Acknowledgments}

This work was supported by U01 MH093765-02, 9P41EB015922-15, 1R01MH094343-01, and P41RR013642. The authors also would like to thank their colleagues at the Martinos Center for Biomedical Imaging at Massachusetts General Hospital.

\section{References}

1. White JG, Southgate E, Thomson JN, Brenner S. Factors that determine connectivity in the nervous system of Caenorhabditis elegans. Cold Spring Harb Symp Quant Biol. 1983; 48(Pt 2):633-640. [PubMed: 6586380]

2. Lichtman JW, Livet J, Sanes JR. A technicolour approach to the connectome. Nat Rev Neurosci. Jun; 2008 9(6):417-422. [PubMed: 18446160]

3. Kasthuri N, Lichtman JW. The rise of the 'projectome'. Nat Methods. Apr; 2007 4(4):307-308. [PubMed: 17396125]

4. Lichtman JW, Sanes JR. Ome sweet ome: what can the genome tell us about the connectome? Curr Opin Neurobiol. Jun; 2008 18(3):346-353. [PubMed: 18801435]

5. Jahanshad, N.; Ryles, A.; McMahon, KL., et al. Discovery of genes that affect human brain connectivity: A genome-wide analysis of the connectome. Paper presented at: International Symposium on Biomedical Imaging; May 2-5, 2012; Barcelona, Spain. 2012.

6. Jahanshad, N.; Kohannim, O.; Toga, AW., et al. Diffusion imaging protocol effects on genetic associations. Paper presented at: International Symposium on Biomedical Imaging; May 2-5, 2012; Barcelona, Spain. 2012.

7. Zhan, L.; Leow, AD.; Aganj, I., et al. Differential Information Content in Staggered Multiple Shell HARDI Measured by the Tensor Distribution Function. Paper presented at: International Symposium on Biomedical Imaging; March 30-April 2, 2011; Chicago, Illinois. 2011.

8. Le Bihan D. Looking into the functional architecture of the brain with diffusion MRI. Nat Rev Neurosci. Jun; 2003 4(6):469-480. [PubMed: 12778119]

9. Beaulieu C. The basis of anisotropic water diffusion in the nervous system - a technical review. NMR Biomed. Nov-Dec;2002 15(7-8):435-455. [PubMed: 12489094]

10. Basser PJ, Mattiello J, LeBihan D. Estimation of the effective self-diffusion tensor from the NMR spin echo. J Magn Reson B. Mar; 1994 103(3):247-254. [PubMed: 8019776]

11. Moseley M, Bammer R, Illes J. Diffusion-tensor imaging of cognitive performance. Brain Cogn. 2002; 50(3):396-413. [PubMed: 12480486]

12. Wedeen VJ, Hagmann P, Tseng WY, Reese TG, Weisskoff RM. Mapping complex tissue architecture with diffusion spectrum magnetic resonance imaging. Magn Reson Med. Dec; 2005 54(6):1377-1386. [PubMed: 16247738]

13. Zhan W, Kang GA, Glass GA, et al. Regional alterations of brain microstructure in Parkinson's disease using diffusion tensor imaging. Movement Disorders. 2012; 27(1):90-97. [PubMed: 21850668]

14. Dennis, EL.; Jahanshad, N.; Toga, AW., et al. Test-retest Reliability of Graph Theory Measures of Structural Brain Connectivity. Paper presented at: 15th International Conference on Medical Image Computing and Computer Assisted Intervention; October 1-5, 2012; Nice, France. 2012. 
15. Lenglet C, Campbell JS, Descoteaux M, et al. Mathematical methods for diffusion MRI processing. Neuroimage. Mar; 2009 45(1 Suppl):S111-122. [PubMed: 19063977]

16. Hagmann P, Cammoun L, Gigandet X, et al. Mapping the structural core of human cerebral cortex. Plos Biol. Jul; 2008 6(7):1479-1493.

17. Xue R, van Zijl PC, Crain BJ, Solaiyappan M, Mori S. In vivo three-dimensional reconstruction of rat brain axonal projections by diffusion tensor imaging. Magnetic resonance in medicine : official journal of the Society of Magnetic Resonance in Medicine / Society of Magnetic Resonance in Medicine. Dec; 1999 42(6):1123-1127. [PubMed: 10571934]

18. Basser PJ, Pajevic S, Pierpaoli C, Duda J, Aldroubi A. In vivo fiber tractography using DT-MRI data. Magnetic resonance in medicine : official journal of the Society of Magnetic Resonance in Medicine / Society of Magnetic Resonance in Medicine. Oct; 2000 44(4):625-632. [PubMed: 11025519]

19. Mori S, Crain BJ, Chacko VP, van Zijl PC. Three-dimensional tracking of axonal projections in the brain by magnetic resonance imaging. Ann Neurol. Feb; 1999 45(2):265-269. [PubMed: 9989633]

20. Jones DK, Travis AR, Eden G, Pierpaoli C, Basser PJ. PASTA: pointwise assessment of streamline tractography attributes. Magnetic resonance in medicine : official journal of the Society of Magnetic Resonance in Medicine / Society of Magnetic Resonance in Medicine. Jun; 2005 53(6): 1462-1467. [PubMed: 15906294]

21. Aganj I, Lenglet C, Jahanshad N, et al. A Hough transform global probabilistic approach to multiple-subject diffusion MRI tractography. Med Image Anal. Aug; 2011 15(4):414-425. [PubMed: 21376655]

22. Wakana S, Jiang H, Nagae-Poetscher LM, van Zijl PC, Mori S. Fiber tract-based atlas of human white matter anatomy. Radiology. Jan; 2004 230(1):77-87. [PubMed: 14645885]

23. Wakana S, Caprihan A, Panzenboeck MM, et al. Reproducibility of quantitative tractography methods applied to cerebral white matter. Neuroimage. Jul 1; 2007 36(3):630-644. [PubMed: 17481925]

24. Shattuck DW, Mirza M, Adisetiyo V, et al. Construction of a 3D probabilistic atlas of human brain structures. NeuroImage. Nov 21; 2008 39(3):1064-1080. [PubMed: 18037310]

25. Tzourio-Mazoyer N, Landeau B, Papathanassiou D, et al. Automated anatomical labeling of activations in SPM using a macroscopic anatomical parcellation of the MNI MRI single-subject brain. NeuroImage. Jan; 2002 15(1):273-289. [PubMed: 11771995]

26. Eickhoff SB, Stephan KE, Mohlberg H, et al. A new SPM toolbox for combining probabilistic cytoarchitectonic maps and functional imaging data. NeuroImage. May 1; 2005 25(4):1325-1335. [PubMed: 15850749]

27. Hua K, Zhang J, Wakana S, et al. Tract probability maps in stereotaxic spaces: Analyses of white matter anatomy and tract-specific quantification. NeuroImage. Jan 1; 2008 39(1):336-347. [PubMed: 17931890]

28. Gerig, G.; Gouttard, S.; Corouge, I. Analysis of brain white matter via fiber tract modeling. Paper presented at: Engineering in Medicine and Biology Society, 2004. IEMBS '04. 26th Annual International Conference of the IEEE; 2004.

29. Maddah M, Zollei L, Grimson WE, Westin CF, Wells WM. A Mathematical Framework for Incorporating Anatomical Knowledge in DT-MRI Analysis. Proceedings / IEEE International Symposium on Biomedical Imaging: from nano to macro. 2008; 4543943:105-108.

30. Li H, Xue Z, Guo L, Liu T, Hunter J, Wong ST. A hybrid approach to automatic clustering of white matter fibers. Neuroimage. Jan 15; 2010 49(2):1249-1258. [PubMed: 19683061]

31. Li Y, Liu Y, Li J, et al. Brain anatomical network and intelligence. PLoS computational biology. May.2009 5(5):e1000395. [PubMed: 19492086]

32. O'Donnell LJ, Westin CF. Automatic tractography segmentation using a high-dimensional white matter atlas. IEEE Trans Med Imaging. 2007; 26(11):1562-1575. [PubMed: 18041271]

33. Xia Y, Turken U, Whitfield-Gabrieli SL, Gabrieli JD. Knowledge-Based Classification of Neuronal Fibers in Entire. Brain. 2005:205-212.

34. Shi Y, Dinov I, Toga A. Cortical Shape Analysis in the Laplace-Beltrami Feature Space. Proc MICCAI. 2009:208-215. 
35. Shi Y, Lai R, Kern K, Sicotte N, Dinov I, Toga AW. Harmonic surface mapping with LaplaceBeltrami eigenmaps. Med Image Comput Comput Assist Interv Int Conf Med Image Comput Comput Assist Interv. 2008; 11(Pt 2):147-154.

36. Shi Y, Morra J, Thompson P, Toga A. Inverse-Consistent Surface Mapping with Laplace-Beltrami Eigen-Features. Proc IPMI. 2009:694-697.

37. Jin, Y.; Shi, Y.; Jahanshad, N., et al. 3D elastic registration improves HARDI-derived fiber alignment and automated tract clustering. Paper presented at: International Symposium on Biomedical Imaging; March 30-April 2, 2011; Chicago, Illinois. 2011.

38. Prasad, G.; Jahanshad, N.; Aganj, I.; Sapiro, G.; Toga, AW.; Thompson, PM. Atlas-based fiber clustering for multi-subject analysis of high angular resolution diffusion imaging tractography. Paper presented at: International Symposium on Biomedical Imaging; March 30-April 2, 2011; Chicago, Illinois. 2011.

39. Bassett DS, Gazzaniga MS. Understanding complexity in the human brain. TRENDS in Cognitive Sciences. 2011; 15(5):200-209. [PubMed: 21497128]

40. Bullmore E, Sporns O. Complex brain networks: graph theoretical analysis of structural and functional systems. Nat Rev Neurosci. Mar; 2009 10(3):186-198. [PubMed: 19190637]

41. Watts DJ, Strogatz SH. Collective dynamics of 'small-world' networks. Nature. Jun 4; 1998 393(6684):440-442. [PubMed: 9623998]

42. Sporns O, Zwi JD. The small world of the cerebral cortex. Neuroinformatics. 2004; 2(2):145-162. [PubMed: 15319512]

43. van den Heuvel MP, Stam CJ, Kahn RS, Hulshoff Pol HE. Efficiency of functional brain networks and intellectual performance. The Journal of neuroscience : the official journal of the Society for Neuroscience. Jun 10; 2009 29(23):7619-7624. [PubMed: 19515930]

44. Gong G, Rosa-Neto P, Carbonell F, Chen ZJ, He Y, Evans AC. Age- and gender-related differences in the cortical anatomical network. The Journal of neuroscience : the official journal of the Society for Neuroscience. Dec 16; 2009 29(50):15684-15693. [PubMed: 20016083]

45. Dennis, EL.; Jahanshad, N.; Toga, AW., et al. Changes in anatomical brain connectivity between ages 12 and 30: A HARDI study of 484 adolescents and adults. Paper presented at: International Symposium on Biomedical Imaging; May 2-5, 2012; Barcelona, Spain. 2012.

46. Shu N, Liu Y, Li J, Li Y, Yu C, Jiang T. Altered anatomical network in early blindness revealed by diffusion tensor tractography. PloS one. 2009; 4(9):e7228. [PubMed: 19784379]

47. Wang L, Zhu C, He Y, et al. Altered small-world brain functional networks in children with attention-deficit/hyperactivity disorder. Human brain mapping. Feb; 2009 30(2):638-649. [PubMed: 18219621]

48. Daianu, M.; Jahanshad, N.; Dennis, EL., et al. Left versus right hemisphere differences in brain connectivity: 4-Tesla HARDI tractography in 567 twins. Paper presented at: International Symposium on Biomedical Imaging; May 2-5, 2012; Barcelona, Spain. 2012.

49. Chiang MC, McMahon KL, de Zubicaray GI, et al. Genetics of white matter development: A DTI study of 705 twins and their siblings aged 12 to 29. Neuroimage. Feb 1; 2011 54(3):2308-2317. [PubMed: 20950689]

50. Prasad, G.; Kohannim, O.; Joshi, S., et al. White Matter Tract Analysis in 454 Adults using Maximum Density Paths. Paper presented at: MICCAI 2011 Workshop on Computational Diffusion MRI; September 18-22, 2011; Toronto, Canada. 2011.

51. Jin, Y.; Shi, Y.; Joshi, S., et al. Heritability of White Matter Fiber Tract Shapes: A HARDI Study of 198 Twins. Paper presented at: MICCAI 2011 Workshop on Multi-Modal Brain Image Analysis (MBIA); September 18-22, 2011; Toronto, Canada. 2011.

52. Dennis EL, Jahanshad N, Toga AW, et al. Abnormal Structural Brain Connectivity in Healthy Carriers of the Autism Risk Gene, CNTNAP2. Brain Connectivity. 2012 in press.

53. Nir, T.; Jahanshad, N.; Jack, CR., et al. Small world network measures predict white matter degeneration in patients with early-stage mild cognitive impairment. Paper presented at: International Symposium on Biomedical Imaging; May 2-5, 2012; Barcelona, Spain. 2012. 